Michael Schulte-Markwort, 2015:

\title{
BURNOUT-KIDS: Wie das Prinzip Leistung unsere Kinder überfordert
}

München: Pattloch-Verlag GmbH \& Co. KG, 269 Seiten, € 19,99

Das Burnout-Syndrom ist vor allem in der Berufstätigkeit von Erwachsenen ein geläufiger Terminus geworden, um auf einen schleichend oder plötzlich einsetzenden Erschöpfungszustand hinzuweisen, der sich körperlich und psychisch manifestiert, durch langandauernde Überforderungen hervorgerufen wird und verschiedene Entwicklungsetappen durchlaufen kann: vom Idealismus und Enthusiasmus bis zur völligen Erschöpfung, Apathie, psychosomatischen Reaktionen und sozialen Auffälligkeiten. Für die Prävention und Bewältigung haben sich u.a. Maßnahmen der Psychohygiene, soziale Unterstützungen und die Supervision bewährt.

Kann Burnout auch bei Kindern und Jugendlichen auftreten? Der Autor der vorliegenden Schrift, ein bekannter Hamburger Arzt für Kinder- und Jugendpsychiatrie und Psychotherapie, ist in den letzten Jahren zu der Erkenntnis gekommen, dass „Burnout bei unseren Kindern angekommen" ist (S. 11). Zuvor war er der Meinung, so sein selbstkritisches Bekenntnis, diese Erkrankung existiere im Kindes- und Jugendalter nicht, und diagnostizierte die betreffenden Symptome teilweise falsch als Hinweise für Depressionen. Er ruft deshalb jetzt alle verantwortlichen Erwachsenen (Eltern, PädagogInnen, ÄrztInnen) dazu auf, sich dem Phänomen Burnout bei Kindern und Jugendlichen zu stellen und für eine effektive Prophylaxe und Behandlung zu sorgen. Seine Forderung: Die Lebensund Lernbedingungen der heranwachsenden Generation sollten von uns sorgfältig analysiert werden, um die Entwicklung von „BurnoutKids" zu verhindern. Das ist zweifellos ein berechtigter Anspruch, auch angesichts seiner Erfahrung, dass etwa zwanzig Prozent der Kinder/Jugendlichen von „seelischen Erkrankungen" betroffen sein können. Die allgemeine Verständlichkeit, Anschaulichkeit und Praxisnähe seiner Darlegungen erleichtern die Lektüre, während der Verzicht auf eine Literaturanalyse das Erfassen des wissenschaftlichen Kontextes etwas erschwert.

Das erste Kapitel („Der Befund") befasst sich sehr differenziert und BURN
KIDS Wie das Prinzip Leistung unsere Kinder überforder Michael Schulte-Markwor

ритосне individuumzentriert an-

hand von fünf Fallbesprechungen mit der Symptomatik des Burnout, vom Verfasser auch als Erschöpfungsdepression bezeichnet (die Depression folgt der Erschöpfung). Überzeugend kann nachgewiesen werden, „dass es sich um sehr individuelle und komplexe Konstellationen handelt, die immer individuell verstanden und angegangen werden müssen" (S. 69), indem die Hintergründe in jedem Einzelfall „,ausgelotet" werden sollten. Schwerpunkte könnten dabei u.a. die Lebensumstände der Kinder/ Jugendlichen, ihre familiäre Situation, die Schullaufbahn, die körperliche und psychische Befindlichkeit und infrage kommende Interventionsmöglichkeiten sein. Auf wichtige extrapersonale Bedingungen der Genese des Störungsbildes wird hingewiesen.

Der Verfasser hebt im zweiten Kapitel („Die Diagnose ${ }^{\prime}$ ) hervor: Die Burnout-Diagnose ist keine Modeerscheinung, vielmehr handelt es sich um ein eigenständiges Krankheitsbild, dessen Symptome auch schon früher bekannt waren. Im Gegensatz zu anderen psychischen Erkrankungen (Schizophrenie, endogene Depressionen, Autismus) ist aber positiv zu konstatieren: Die Erschöpfungsdepression kann verhindert werden, wenn rechtzeitig und angemessen darauf reagiert wird. Dabei sind aber, wie gezeigt werden kann, vielfältige diagnostische Anforderungen zu erfüllen, wobei nicht nur unterschiedliche Verfahren, sondern auch internationale Klassifikationssysteme (ICD-10, DSM V) hilfreich sein können. Interessante Informationen erfolgen zur Häufigkeit psychi- 
scher Erkrankungen im Kindes- und Jugendalter (Burnout 3-5\%, mehrheitlich Mädchen), über typische Symptome der Erschöpfungsdepression, Angst und Stress.

Um Burnout vorzubeugen oder adäquate Hilfe zu gewähren, wenn die Charakteristika der Erschöpfungsdepression in Erscheinung treten, ergibt sich die Frage, welche Ursachen dafür verantwortlich zu machen sind. Sicher ist es richtig, hier nicht monokausal vorzugehen, sondern multifaktorielle Konstellationen - vgl. Kapitel „Die Ursachen“ - in Betracht zu ziehen. Der Autor verweist auf ein "Puzzle“, das den Boden bereitet für Erschöpfung und Depression: historische und gesellschaftliche Facetten sowie individuelle und kollektive Prozesse. Aufschlussreiche Ausführungen werden gemacht zum Burnout als „Familienerbe“, Familien in der "Ökonomiefalle" und Risikofaktoren im Lebensumfeld der Kinder und Jugendlichen. Nicht zu Unrecht wird auf die schulbezogene Dynamik verwiesen, die zu Burnout führt, insbesondere das Gymnasium (Prototyp für Schule) genannt, das eine Erschöpfungsdepression auslösen kann. Nicht zuletzt das in allen Lebensbereichen dominierende Leistungsprinzip ist kritisch zu hinterfragen.

Was kann getan werden, damit ein Kind oder ein/e Jugendliche/r nicht zum "Burnout-Kid" wird? Mit dieser Frage setzt sich das vierte Ka- pitel („Die Behandlung“) gründlich und überzeugend auseinander. Wichtige Hinweise erfolgen zum Einsatz von Medikamenten (Antidepressiva) und zur Psychotherapie (Verhaltenstherapie, tiefenpsychologische Verfahren). Wertvolle Ratschläge werden zu den in den Familien gelebten Werten (Fürsorge, fördern ohne zu überfordern, verlässliche Beziehungsgestaltung, Verbesserung der individuellen Widerstandsfähigkeit durch Entspannung) unterbreitet. Vor allem gilt: In der Gesellschaft, in der Familie, in der Schule und in anderen Gemeinschaften sollte verantwortungsbewusst mit dem Leistungsprinzip umgegangen, Überforderungen vermieden und die Sorgen der Mädchen und Jungen ernst genommen werden.

Alles in allem: Eine notwendige und anregende Publikation, der ein konstruktiver wissenschaftlicher Disput zum Wohle aller Kinder zu wünschen ist, damit diese gesund und ohne Burnout aufwachsen können. Sie sensibilisiert für gesellschaftliche und familiäre Defizite (maximale Ökonomisierung, falsche Werte) und fordert insbesondere Eltern, PädagogInnen und ÄrztInnen auf, den Gründen für das „Ausbrennen" der Kinder und Jugendlichen nachzugehen und ihnen bewusst und zielgerichtet zu begegnen.

Dr. habil. W. Topel, Leipzig

DOI 10.2378/uj2015.art63d 